\title{
Reversed phase HPLC for strontium ranelate: Method development and validation applying experimental design
}

BÉLA KOVÁCS $S^{1,2}$ *

LAJOS KRISTÓF KÁNTOR ${ }^{1}$

MIRCEA DUMITRU CROITORU ${ }^{3}$

ÉVA KATALIN KELEMEN ${ }^{1}$

MONA OBREJA

ELŐD ERNŐ NAGY ${ }^{2}$

BLANKA SZÉKELY-SZENTMIKLÓSI ${ }^{4}$

ÁRPÁD GYÉRESI ${ }^{4}$

${ }^{1}$ Gedeon Richter Romania

540306, Tîrgu Mureș, Romania

${ }^{2}$ Department of Biochemistry and Environmental Chemistry, Faculty of Pharmacy, University of Medicine and Pharmacy, 540139, Tîrgu Mureș, Romania

${ }^{3}$ Department of Toxicology and Biopharmacy Faculty of Pharmacy, University of Medicine and Pharmacy, 540139, Tîrgu Mureș

Romania

${ }^{4}$ Department of Pharmaceutical Chemistry Faculty of Pharmacy, University of Medicine and Pharmacy, 540139, Tîrgu Mureș

Romania

Accepted January 18, 2018

Published online February 19, 2018

\begin{abstract}
A reverse-phase HPLC (RP-HPLC) method was developed for strontium ranelate using a full factorial, screening experimental design. The analytical procedure was validated according to international guidelines for linearity, selectivity, sensitivity, accuracy and precision. A separate experimental design was used to demonstrate the robustness of the method. Strontium ranelate was eluted at 4.4 minutes and showed no interference with the excipients used in the formulation, at $321 \mathrm{~nm}$. The method is linear in the range of $20-320 \mu \mathrm{g} \mathrm{mL}^{-1}\left(R^{2}=0.99998\right)$. Recovery, tested in the range of $40-120 \mu \mathrm{g} \mathrm{mL}^{-1}$, was found to be 96.1-102.1 \%. Intra-day and intermediate precision RSDs ranged from 1.0-1.4 and 1.2-1.4 \%, resp. The limit of detection and limit of quantitation were 0.06 and $0.20 \mu \mathrm{g} \mathrm{mL}^{-1}$, resp. The proposed technique is fast, cost-effective, reliable and reproducible, and is proposed for the routine analysis of strontium ranelate.
\end{abstract}

Keywords: strontium ranelate, HPLC, experimental design, full factorial design

Strontium ranelate $\left(\mathrm{SrR}\right.$, Protelos $^{\circledR}$, Osseor $\left.^{\circledR}\right)$ behaves as a dual acting bone agent by inhibiting osteoclast activity and at the same time promoting osteoblast differentiation and maturation (1). Although several controversies emerged regarding the risk/benefit ratio, as a result communicated by the Pharmacovigilance Risk Assessment Committee (PRAC) of the European Medicine Agency in April 2014, SrR remained in therapy, with further restrictions (2), as a cost-effective alternative for the treatment of osteoporosis in both male and female patients.

Structurally, SrR is a di-strontium salt of 2-(2-carboxy-4-cyano-5-[N,N-di(carboxymethyl)amino]thiophene-3-yl) acetic acid (ranelic acid) (Fig. 1).

\footnotetext{
*Correspondence; kovacsbela89@gmail.com; kovacs.bela@umftgm.ro
} 


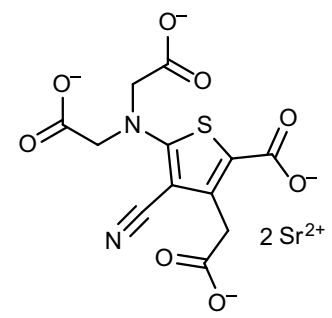

Fig. 1. Chemical structure of strontium ranelate.

Literature data of the active substance revealed that only a few analytical methods have been developed for the assay of strontium ranelate, including HPLC (3-5), HPTCL (6), UV spectrophotometry $(7,8)$, capillary electrophoresis (9) and others (10, 11).

The up-to-date "quality by design" approach has been successfully used in the pharmaceutical development and characterisation of tablets, optimization of tablet formulations $(12,13)$ and also for the analytical method development and validation of HPLC (14, 15) and other analytical methods (16). Design of experiments (DoE) has many advantages in pharmaceutical drug formulations and analytical method development and validation in comparison with techniques like changing one factor at a time (COST). While traditional developmental approaches are mainly empirical and are often conducted using the COST method, DoE implies the possibility of performing systematic and multivariate experiments in order to fully understand the process and product characteristics (17-19). The reported HPLC methods (3-5) use a multicomponent solvent system and laborious sample preparation as well as conventional method development and validation.

The main goal of the present study was to develop a high throughput HPLC method for quality control of strontium ranelate, which fulfils method validation requirements requested by international guidelines (20). Also, we investigated the effectiveness of a computational design approach for method development and validation.

\section{EXPERIMENTAL}

\section{Reagents}

The active pharmaceutical ingredient SrR octahydrate $(22.5 \%$ water determined by the Karl-Fischer titrimetric method, $\gamma=99.2 \%$ by HPLC) was from Dishman Pharmaceuticals and Chemicals, India. Methanol (MeOH) and trifluoroacetic acid (TFA) used were from Merck, Germany. Mannitol (Pearlitol 300DC, Ph. Eur., Roquette Pharma, France), maltodextrin (Lycatab DSH, Ph. Eur., Roquette Pharma) and aspartame (Ph. Eur., Sigma

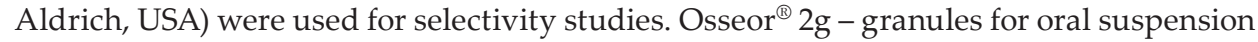
(Lés Laboratoires Servier, France) was purchased from a local pharmacy.

\section{Preparation of standard solution}

For the stock solution, $8 \mathrm{mg}$ of SrR octahydrate was weighed in a $100-\mathrm{mL}$ volumetric flask and made up with TFA $0.1(0.1 \%, V / V)$ to the mark. The final solution was stirred on 
an ultrasound bath for 2 min and filtered through $0.45-\mu \mathrm{m}$ Whatman ${ }^{\circledR}$ nylon filters (General Electric Healthcare, UK) in brown HPLC vials. The first $2 \mathrm{~mL}$ of the filtered solution were discarded.

\section{Chromatographic conditions and apparatus}

HPLC separations were performed with an Agilent 1260 Infinity system with OpenLAB CDS software (Agilent Technologies, USA), using a RP-HPLC column, Inertsil ODS-3, $4.6 \times 250 \mathrm{~mm}, 5 \mu \mathrm{m}$ porosity (GL Sciences, Japan). The optimized isocratic mobile phase was composed of $\mathrm{MeOH}$ and TFA $(0.1 \%, V / V)$ in the proportion 52:48 $(V / V)$. The samples $(20 \mu \mathrm{L})$ were injected using an auto sampler and a quaternary pump with degasser. Signal detection was carried out with a diode array detector at $321 \mathrm{~nm}$. All analyses were performed at $34{ }^{\circ} \mathrm{C}$.

\section{Method optimization using design of experiments (DoE)}

Experimental design. - In order to optimize the mobile phase composition and ambient conditions of the HPLC system, we performed a screening type, full factorial design (optimization) with three centre points using MODDE 11.0 software (Umetrics, Sweden). The factors defined in the current model are as follows: mobile phase composition, flow rate and column temperature. Taking into consideration that the mobile phase composition and flow rate were defined at three levels, and column temperature at two levels, the full factorial design returned 21 experiments $\left(3^{2}=9\right.$ runs in our case, where 3 represents the levels defined, and 2 in the exponent stands for the number of factors. Respective experiments were performed for both lower and higher temperature settings, yielding 18 runs, and resulting in a total of 21 runs with centre pointed experiments). Experiments were run in replicate samples and peak asymmetry, theoretical plate numbers and retention times being noted as responses (Table I).

Table I. Selected factors and responses for full factorial, screening experimental design

\begin{tabular}{lccc}
\hline \multicolumn{4}{c}{ Factors used in the experimental design } \\
\hline Level applied & -1 & 0 & +1 \\
$\begin{array}{l}\text { Mobile phase composition } \\
(\text { methanol proportion, } \%)\end{array}$ & 20 & 50 & 90 \\
Flow rate $\left(\mathrm{mL} \mathrm{min}^{-1}\right)$ & 0.5 & 1.0 & 1.5 \\
Column temperature $\left({ }^{\circ} \mathrm{C}\right)$ & 30 & 40 & 50 \\
\hline & Responses defined for experimental evaluation & \\
\hline Requirement & Min & Target & Max \\
Peak asymmetry & 0.8 & 1 & 1.5 \\
Theoretical plate number $(N)^{\mathrm{a}}$ & 1000 & - & - \\
Retention time $(\mathrm{min})$ & 3 & 5 & 7 \\
\hline
\end{tabular}

${ }^{a}$ Defined per column length. 
Model fitting. - The model was fitted using the partial least squares (PLS) method. The experimental model was characterised based on the significance of the regression model using the analysis of variance (ANOVA) and its lack of fit test, and the model performance indicators.

Evaluation of experimental design. - The summary of fit plot represents the model performance indicators: the leftmost bar represents the goodness of fit $\left(R^{2}\right)$, being an indicator of how well the regression model can be made to fit raw data. Taking into account that $R^{2}$ alone is not sufficient to evaluate the validity of a model, goodness of predictability - second bar - $\left(Q^{2}\right)$ is a useful indicator and estimates the predictive power of the model. In general, a model is judged as good if the difference between goodness of fit $\left(R^{2}\right)$ and goodness of predictability $\left(Q^{2}\right)$ is lower than $0.2-0.3$, otherwise indicating an inappropriate and insignificant model, and at the same time it is desirable for $Q^{2}$ values to be higher than 0.5 . The third and rightmost bars represent model validity and reproducibility, resp. Model validity describes the appropriateness of the selected type of model in general sense and is accepted if it returns a reference value greater than 0.25 (17). Taking into consideration that when the pure error is very small (replicate results almost identical), model validity can be low even though the model is good and complete (18). Finally, model reproducibility gives information about the control of the experimental procedure; the higher the numerical value (>0.5), the smaller is the replicate (pure) error. Also, evaluation of raw data should be performed on the basis of the coefficient plots of selected responses. Coefficient plots represent the dominating factors of the responses and the direction in which the influence is powerful. Based on the coefficient plots, model refinement is performed by deleting insignificant factors to response interactions $(p>0.05)(17,19,21)$.

Optimization. - After model fitting and refinement, an optimizer run was performed and factor settings were defined using the prediction spreadsheet function.

\section{Validation of the optimized analytical method}

The developed method based on the DoE optimizer was validated according to international guidelines (20).

Linearity. - Linearity was investigated in the range of $20-320 \mu \mathrm{g} \mathrm{m}^{-1}$ at 11 points $(20$, $40,60,70,80,90,100,120,160,240,320 \mu \mathrm{g} \mathrm{mL}^{-1}$ ) repeated five times for each concentration. Solutions were prepared from the stock solution of $400 \mu \mathrm{g} \mathrm{mL} \mathrm{mL}^{-1}$.

Selectivity. - Selectivity studies were performed according to the qualitative and quantitative composition of the original product, Osseor ${ }^{\circledR} 2$ g-granules for oral suspension. The pharmaceutical formulation was prepared from mannitol $4.0 \mathrm{~g}$, maltodextrin $0.4 \mathrm{~g}$ and aspartame $0.02 \mathrm{~g}$ per dose. Samples containing individual excipients, a placebo mixture and placebo spiked with SrR were prepared and analysed under the same conditions (dissolved in TFA $0.1 \%, V / V$, and using the same mobile phase).

Accuracy (recovery). - Accuracy testing was performed using placebo samples spiked with $\operatorname{SrR}(50,75,100,125$ and $150 \%$ of the working concentration, repeated three times for each concentration).

Robustness. - Method robustness was tested using a separate experimental design (Plackett-Burman model fitted with multiple linear regression, MLR) with three centre points. The Plackett-Burman (PB) design is an orthogonal two-level experimental design, which 
allows to test a large number of factors, but no factor interactions can be evaluated. Taking into consideration that robustness testing implies linear analysis of the factors and responses, the PB model represents one of the commonly used designs for method robustness testing. After model fitting and raw data evaluation, various outcomes of robustness testing are to be considered on the basis of the $R^{2}$ and $Q^{2}$ values: $i$ ) inside specification and significant model, ii) inside specification and insignificant model, iii) outside specification and significant model, iv) outside specification and insignificant model. The second limiting case (results inside specification and insignificant model) represents the optimal outcome of robustness testing, since all results are situated in-between the specification limits and no significant relationship is observed between the factors and responses (17). The factors defined for the experimental design were the same as described in DoE optimization, with the following intervals: methanol proportion $50-54 \%(V / V)$, flow rate $0.65-0.75 \mathrm{~mL} \mathrm{~min}^{-1}$, column temperature $32-36{ }^{\circ} \mathrm{C}$. The investigated factors were defined at two levels, resulting in 11 experiments. The experiments were run in two replicates, and the responses were the same as defined in optimization DoE, respecting the corresponding intervals in all three cases.

Furthermore, method robustness was tested for individual changes of the detection wavelength (319 and $323 \mathrm{~nm}$ ) and filter type (regenerated cellulose $0.22-\mu \mathrm{m}$ filter $v$ s. routine analysis filter).

Limit of detection (LOD) and limit of quantitation (LOQ). - Limit of detection (LOD) and limit of quantitation $(L O Q)$ were defined as concentrations at a signal-to-noise ratio of 3:1, for six replicate determinations, and at a signal-to-noise ratio of 10:1, for three replicate determinations.

Precision. - Samples containing Osseor ${ }^{\circledR}$ granules, corresponding to $6.24 \mathrm{~g}$ SrR anhydrous, were prepared and analysed six times on a single day and on different days by two analysts.

\section{Statistical analysis}

Statistical analyses were performed using Minitab 17 software (Minitab Inc., State College, PA, USA) and Statistica 8.0 software (StatSoft, Tulsa, OK, USA).

Outlier values from the experimental designs were detected using Grubb's test for outliers at a significance level of $\alpha=0.05(p<0.05)$.

Normal distribution of the residuals for linearity testing was demonstrated by the Shapiro-Wilk test (confidence interval of $95 \%$ ), and the normality test for residuals was also assessed by a Q-Q plot. The test was considered significant if the $W$ value calculated for the analyte was higher than the critical tabulated value and $p<0.05$, simultaneously. Linearity acceptance was also assessed, based on the relative standard deviation of subsequent injections for each concentration (requirement: $<2 \%$ ), and the plot of response versus the corresponding concentration values was calculated with an acceptance limit of $2.5 \%$ of the target level response factor. Calibration curve significance was tested with ANOVA $F$-test and its test for lack of fit (confidence limit of $95 \%$ ). Process capability was calculated for the plot of response vs. concentration values in order to assess the ability of the process to perform within the specification limits (acceptance value $C_{\mathrm{pk}}>1.33$ ).

Student's $t$-test was used in order to statistically compare the result sets of intra-day and intermediate precision, statistical significance being set at a confidence level of $95 \%$ (22). 


\section{RESULTS AND DISCUSSION}

\section{Screening experimental design (optimization)}

Two experiments were excluded from this procedure due to inappropriate retention times; outlier values were excluded from the design as well. The results are presented in Table II.

Table II. Optimization DoE experimental runs - conditions set and raw analysis results

\begin{tabular}{|c|c|c|c|c|c|c|c|}
\hline 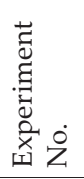 & $\Xi \begin{array}{c}\overrightarrow{0} \\
\check{0}\end{array}$ & 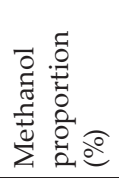 & 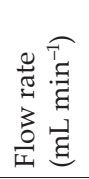 & 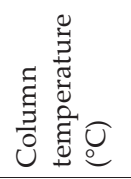 & 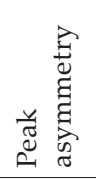 & 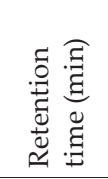 & Z \\
\hline $1^{\mathrm{a}}$ & 21 & 20 & 0.5 & 30 & - & $27.350^{\mathrm{b}}$ & - \\
\hline 2 & 14 & 50 & 0.5 & 30 & 1.450 & 6.550 & 6747.0 \\
\hline 3 & 1 & 90 & 0.5 & 30 & 0.845 & 5.865 & 319.5 \\
\hline 4 & 6 & 20 & 1 & 30 & 1.150 & $15.730^{\mathrm{b}}$ & 966.0 \\
\hline 5 & 11 & 50 & 1 & 30 & 1.260 & 3.285 & 4790.5 \\
\hline 6 & 18 & 90 & 1 & 30 & 0.655 & 3.085 & 836.0 \\
\hline $7^{a}$ & 13 & 20 & 1.5 & 30 & - & - & - \\
\hline 8 & 4 & 50 & 1.5 & 30 & 1.210 & 2.190 & 3613.5 \\
\hline 9 & 17 & 90 & 1.5 & 30 & 1.215 & 1.885 & 532.5 \\
\hline 10 & 16 & 20 & 0.5 & 50 & 1.230 & $18.35^{\mathrm{b}}$ & 2259.0 \\
\hline 11 & 15 & 50 & 0.5 & 50 & 1.340 & 6.030 & 6135.5 \\
\hline 12 & 2 & 90 & 0.5 & 50 & 0.985 & 5.585 & 314.5 \\
\hline 13 & 19 & 20 & 1 & 50 & 1.160 & 9.440 & 7357.5 \\
\hline 14 & 5 & 50 & 1 & 50 & 1.210 & 2.995 & 4318.5 \\
\hline 15 & 10 & 90 & 1 & 50 & $2.615^{\mathrm{b}}$ & 2.710 & 340.0 \\
\hline 16 & 12 & 20 & 1.5 & 50 & 1.160 & 6.060 & 5545.0 \\
\hline 17 & 3 & 50 & 1.5 & 50 & 1.155 & 1.990 & 3388.0 \\
\hline 18 & 7 & 90 & 1.5 & 50 & 0.925 & 1.880 & 578.5 \\
\hline 19 & 8 & 50 & 1.0 & 40 & 1.305 & 3.120 & 4948.0 \\
\hline 20 & 20 & 50 & 1.0 & 40 & 1.255 & 3.125 & 5073.5 \\
\hline 21 & 9 & 50 & 1.0 & 40 & 1.295 & 3.120 & 4815.5 \\
\hline
\end{tabular}

$N$ - number of theoretical plates per column length.

a Excluded experiments: experiments No. 1 and No. 7 were excluded, because the active substance under the set of chromatographic conditions showed inappropriate retention time.

${ }^{\mathrm{b}}$ Outlier values. 
a)

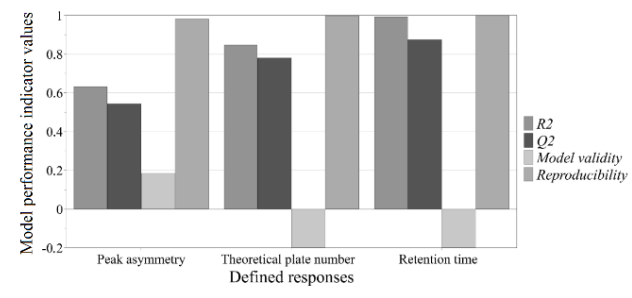

c)

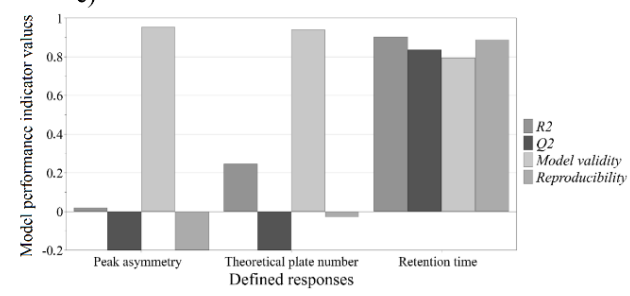

b)

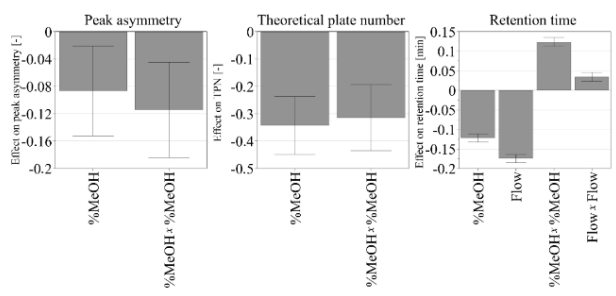

d)

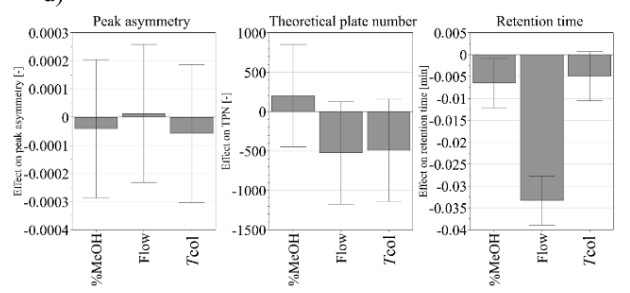

Fig. 2. a) Summary of the fit of screening DoE (optimization), b) correlation plot of responses of screening DoE (optimization), c) summary of the fit of Plackett-Burman model (robustness testing), d) correlation plot of responses of Plackett-Burman model (robustness testing).

For all three responses (peak asymmetry, retention time, theoretical plate number), significant regression models were obtained $(p<0.05)$ and lack of fit was observed $(p<0.05)$ in some cases. This phenomenon is also sustained by low model validity for all responses $(<0.25)$. In contrast to low model validity, model reproducibility showed high values $(\approx 0.99)$ for all responses, since the replicate runs (Table II, exp. nos. 19-21) returned almost identical results, meaning that pure error inside the model was very low, as desirable for replicate HPLC injections. As the pure error inside the model tends to zero, low model validity is explained by the high reproducibility of experimental runs. Moreover, extremely good reproducibility $(>0.9)$ is advantageous in HPLC screening designs, in comparison with model validity, as lower reproducibility results (usually $<0.5$ ) indicate a large pure error inside the model and poor control of the experimental procedure.

The other model performance indicators represent a good model, $R^{2}$ values ranging from 0.63 to 0.99 and $Q^{2}$ values greater than 0.5 (0.54-0.87) (Fig. 2a).

Peak asymmetry is strongly influenced by methanol proportion in the mobile phase, this being inversely proportional to the value of peak asymmetry. Similarly, the theoretical plate number shows a negative correlation with the quantity of methanol in the mobile phase. Methanol proportion and flow rate had a powerful impact on retention time; inverse proportionality was found (Fig. 2b). Also, quadratic terms of the methanol proportion $(\% \mathrm{MeOH} \times \% \mathrm{MeOH})$ had a significant impact on all responses, flow rate (flow $\times$ flow) influencing only retention time in a significant manner. As a consequence, these quadratic terms were retained during model fitting, since their deletion would result in model performance indicator $\left(R^{2}, Q^{2}\right)$ deterioration. Furthermore, the presence of these quadratic 
B. Kovacs et al:: Reversed phase HPLC for strontium ranelate: Method development and validation applying experimental design, Acta Pharm. 68 (2018) 171-183.

Table III. Optimized chromatographic conditions based on the experimental design, and predicted results for the proposed settings

\begin{tabular}{ccc}
\hline \multicolumn{3}{c}{ Optimized chromatographic conditions according to DoE } \\
\hline Methanol (\%) & Flow rate $\left(\mathrm{mL} \mathrm{min}^{-1}\right)$ & Column temperature $\left({ }^{\circ} \mathrm{C}\right)$ \\
\hline 52 & 0.7 & 34 \\
\hline & Predicted results \\
\hline Peak asymmetry & Retention time (minutes) & Theoretical plate number $(N)^{\mathrm{a}}$ \\
1.27 & 4.42 & 4588 \\
$(1.18-1.36)$ & $(4.26-4.59)$ & $(3244-6489)$ \\
\hline
\end{tabular}

Values in brackets indicate the $95 \%$ confidence intervals around the predicted response values.

${ }^{a} \mathrm{~N}$ defined per column length.

terms indicates a non-linear blending of the effects, and the presence of a curved surface inside the model.

After model fitting, the samples were injected five times using the parameters found as the optimal set provided by the software. The conditions set, using the prediction spreadsheet function are displayed in Table III.

The results obtained after five successive injections for the optimal set of chromatographic conditions showed a good correlation with the predicted values, the theoretical plate number being the farthest from the predicted values, but still lying inside the foreseen interval. Under optimized conditions, the analyte eluted at the retention time around $4.4 \mathrm{~min}$ (mean $4.42 \mathrm{~min}, \mathrm{RSD}=0.4 \%$ ) as predicted by the software (Fig. 3b). Peak asymmetry resulted in the mean value of $1.32(\mathrm{RSD}=1.4 \%)$, being marginally higher than the predicted value. The theoretical plate number resulted in $5755(\mathrm{RSD}=2.4 \%)$, a value higher by 1167 compared to the value predicted by the software.

\section{Validation of optimized chromatographic method}

Stability of the stock solution. - Stability of the active pharmaceutical ingredient in the solution (TFA $0.1 \%, V / V$ ) was followed for 168 hours. Degradation of the active substance was $\leq 2 \%$ after 8 hours, reaching approx. $30 \%$ decomposition after the target test period.

Selectivity. - The performed selectivity studies show that SrR could be identified as a sharp, well distinct peak at $321 \mathrm{~nm}$, with no interference from the excipients (Fig. 3c). In the case of mannitol and aspartame, no peak was observed at the proposed detection wavelength, while maltodextrin showed a small peak at $3.71 \mathrm{~min}$ (Fig. 3a), with resolution of 2.71 to SrR.

Linearity. - The method was found to be linear in the range of $20-320 \mu \mathrm{g} \mathrm{mL}^{-1}$, with the coefficient of determination $R^{2}>0.99998$. Relative standard deviation for each concentration was $<1.5 \%$ for replicate injections, and the plot of response values referring to the target concentration was $<1.0 \%$. Statistical analysis revealed that the residuals followed 
a)

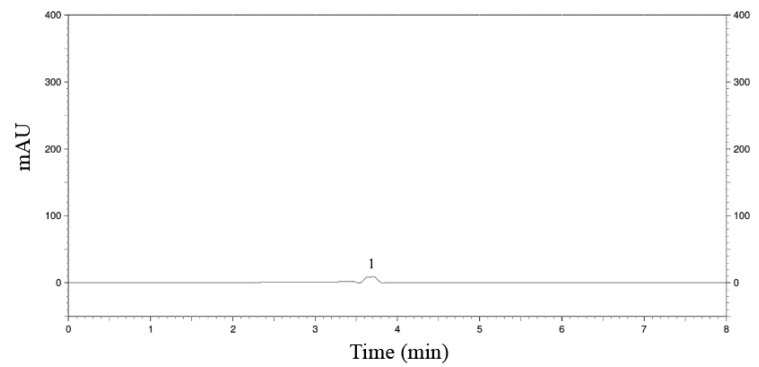

b)

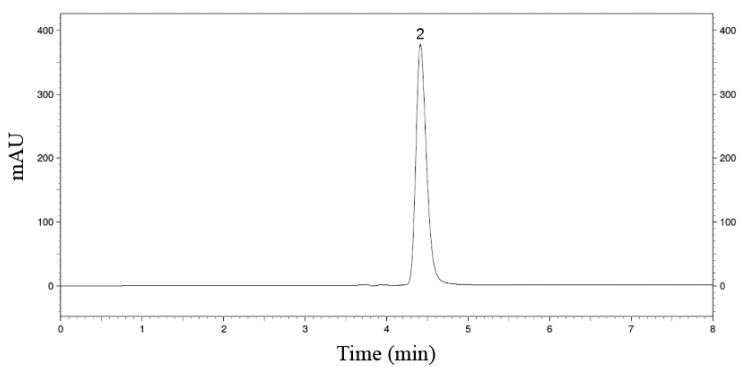

c)

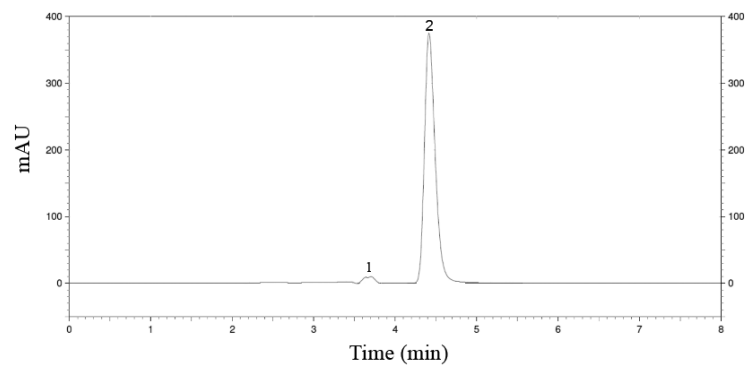

Fig. 3. Typical chromatograms of: a) placebo formulation, b) strontium ranelate from bulk, c) strontium ranelate spiked sample. Key for the peaks: $1-$ maltodextrin $\left(t_{\mathrm{R}}=3.71 \mathrm{~min}\right), 2-$ Sr-ranelate $\left(t_{\mathrm{R}}=4.41 \mathrm{~min}\right)$.

normal distribution, since the obtained $W$-value for strontium ranelate was superior to the critical tabulated value for the Shapiro-Wilks test $\left(W_{\mathrm{SrR}}>W_{a^{\prime}} p>0.05\right)$. Moreover, a strong linear relationship between the responses and predictors $\left(F_{\mathrm{SrR}}>F_{c^{\prime}} p<0.05\right)$ and no lack-offit was observed inside the regression model $\left(F_{\mathrm{SrR}}<F_{\mathrm{c}^{\prime}} p>0.05\right)$, since the $F$-values for strontium ranelate have greater values for relationship testing and smaller values for lackof-fit testing in comparison with the critical tabulated values for the ANOVA F-test and its lack-of-fit test, resp. The process capability assessed for the calculated plot of response values vs. concentration is greater than the acceptance limit of 1.33, indicating that the output of the model (responses vs. predictors) is well controlled and within limits. Statistical results are given in Table IV. 
B. Kovacs et al.: Reversed phase HPLC for strontium ranelate: Method development and validation applying experimental design, Acta Pharm. 68 (2018) 171-183.

Table IV. Validation parameters for HPLC determination of strontium ranelate

\begin{tabular}{|c|c|c|}
\hline Parameter & Result & Statistical results \\
\hline Linearity $\left(\mu \mathrm{g} \mathrm{mL} \mathrm{L}^{-1}\right)$ & $20-320$ & $\begin{array}{c}R^{2}=0.99998 \\
W_{\mathrm{SrR}}=0.949(p=0.63)^{\mathrm{a}} \\
F_{\mathrm{SrR}}=277,769.94(p<0.05)^{\mathrm{b}} \\
F=0.29(p=0.975)^{\mathrm{c}} \\
C_{\mathrm{pk}}=2.47^{\mathrm{d}}\end{array}$ \\
\hline Accuracy $(\%)^{\mathrm{e}}$ & $99.1 \pm 1.7$ & $R^{2}=0.998$ \\
\hline Intra-day precision $(\mathrm{RSD}, \%)^{\mathrm{f}, \mathrm{g}}$ & $1.0-1.4$ & $\begin{aligned} & t_{\text {<analyst } 1 \text { day } 1>}=0.225(p=0.826) \\
& t_{\text {<analyst } 1 \text { day } 2>}=0.04(p=0.969) \\
& t_{\text {<analyst } 2 \text { day } 2>}=0.02(p=0.986)\end{aligned}$ \\
\hline Inter-day precision $(\mathrm{RSD}, \%)^{\mathrm{f}, \mathrm{h}}$ & $1.2-1.44$ & $\begin{array}{c}t_{<\text {analyst } 1 \text { day } 1 \text { vs. day } 2>}=0.770(p=0.452) \\
t_{<\text {analyst } 1 \text { vs. analyst } 2>}=1.250(p=0.226)\end{array}$ \\
\hline Instrument precision (RSD, \%) ${ }^{\mathrm{e}}$ & 0.1 & \\
\hline$L O D\left(\mu g \mathrm{~mL}^{-1}\right)$ & 0.06 & \\
\hline$L O Q\left(\mu \mathrm{g} \mathrm{mL}^{-1}\right)$ & 0.20 & \\
\hline
\end{tabular}

${ }^{a}$ Critical tabulated value of Shapiro-Wilk's test: $W_{\mathrm{a}}=0.850\left(W_{\mathrm{SrR}}-\right.$ Shapiro-Wilk's test results for strontium ranelate).

${ }^{\mathrm{b}}$ Critical tabulated value of ANOVA $F$-test: $F_{\mathrm{c}}=2.649$ ( $F_{\mathrm{SrR}}-$ ANOVA $F$-test results for strontium ranelate).

${ }^{\mathrm{c}}$ Critical tabulated value of ANOVA $F$-test for lack-of-fit: $F_{\mathrm{c}}=2.14$.

${ }^{\mathrm{d}} C_{\mathrm{pk}}>1.33\left(C_{\mathrm{pk}}-\right.$ process capability index $)$.

e $n=5$.

${ }^{\mathrm{f}} n=6$.

g Critical value of $t=2.228, \mathrm{df}=10$.

${ }^{\mathrm{h}}$ Critical value of $t=2.074, \mathrm{df}=22$.

Accuracy (recovery). - Recovery varied from 96.1 to $102.1 \%$ in the tested range. Linearity of the spiked samples was also verified, showing a good linear correlation with $R^{2}=0.998$. Recovery was found to be in the range of $98.2-101.3 \%$ for concentration levels from $80-120 \%$ of the working concentration.

Robustness. - The method proved to be robust in the tested range, with low goodness of fit $\left(R^{2}=0.02-0.24\right)$ and negative goodness of predictability $\left(Q^{2}=-0.49\right.$ to -0.22$)$ values, indicating that minor changes in factor settings did not influence the responses (peak asymmetry and theoretical plate numbers) in a significant manner, the results being within the specification limits under the tested factor settings.

Furthermore, the negative goodness of predictability indicated that the software could not foresee conspicuous variations in results for minor changes in factor settings. This was due to homogenous results of the responses under the tested factor intervals.

In contrast to the DoE performed for factor screening, model validity bars were $>0.5$ for all three responses (peak asymmetry, theoretical plate number, retention time), indicating that the method had a good validity in the proposed setting range (Fig. 2c).

Peak asymmetry and theoretical plate number were not influenced by minor changes in mobile phase composition, column temperature and flow rate. However, retention time was sensitive to minor factor setting modifications, showing a strong negative correlation 
with the proportion of methanol in the mobile phase and the applied flow rate (Fig. $2 \mathrm{~d}$ ). These observations are in accord with the results of DoE screening used for optimization of the method.

The tested changes in sample preparation (use of regenerated cellulose filter) and signal detection had no significant impact on the chromatographic results; peak asymmetry, retention time, and theoretical plate numbers were not influenced by the investigated factors.

Precision. - Method precision was assessed using Osseor ${ }^{\circledR}$ granules for oral suspension. The intra-day and intermediate precision showed low variability between samples, RSD values being less than $2 \%$ for all samples. The performed Student's $t$-test revealed that there was no statistically significant difference between the result sets obtained by the same analyst on the same day and inter-day, nor between two analysts on the same day, indicating that the method provided good intra-day and intermediate precision (Table IV).

Limit of detection and limit of quantitation. - LOD and LOQ for strontium ranelate, expressed for the anhydrous form, were 0.06 and $0.20 \mu \mathrm{g} \mathrm{mL} \mathrm{L}^{-1}$, resp.

\section{Preliminary method applicability}

The assay of strontium ranelate in Osseor ${ }^{\circledR}$ granules resulted in $99.18 \%$ of active substance according to the label claim, with good precision (RSD $<2 \%$ ).

\section{Method benefits}

Unlike the disclosed methods already available for the assay of strontium ranelate, the proposed novel method has shorter sample preparation time, thus offering a high throughput of sample analysis.

This assay works within a wider linearity range and shows superior $L O D$ and $L O Q$ values. Furthermore, signal detection is carried out at $321 \mathrm{~nm}$, where no interference was observed with the excipients present in the original product composition.

\section{CONCLUSIONS}

The suggested method turned out to be an easy, rapid and cost-effective analytical tool for assaying strontium ranelate in bulk samples and, as expected, in pharmaceutical dosage forms. A computational approach to the design of experiments was used to gain as much knowledge about the chromatographic system as possible. The applied experimental designs for method optimization and robustness testing proved to be adequate and can be used for analytical method development. Furthermore, the method proved to meet all validation requirements (linearity, accuracy, sensitivity, robustness, precision) requested by most international guidelines. In addition, the computational method development offered extensive knowledge about the HPLC system, including the possibility to gather in silico information about the responses that characterise the developed analytical method by modifying factor settings. 


\section{REFERENCES}

1. J. Y. Reginster, A. Neuprez, N. Dardenne, C. Beaudart, P. Emonts and O. Bruyère, Efficacy and safety of currently marketed anti-osteoporotic medications, Best Pract. Res. Clin. Endocrinol. Metab. 28 (2014) 809-384; https://doi.org/10.1016/j.beem.2014.09.003

2. European Medicines Agency, Protelos/Osseor to Remain Available But With Further Restrictions, London, February 2014; http://www.ema.europa.eu/docs/en_GB/document_library/Press_release/2014/02/ WC500161971.pdf; last access date April 29, 2017

3. K. Mythili, S. Gayatri, K. R. Teja, K. Chitra and C. U. M. Reddy, Development and validation of RP-HPLC method for the estimation of strontium ranelate in sachet, Int. J. Pharm. Bio. Sci. 2 (2011) 258-263.

4. B. R. C. S. Reddy and N. V. B Rao, A validated stability indicating RP-HPLC method for the determination of strontium ranelate a dual acting bone agent in bulk and sachet dosage form, Rasayan J. Chem. 7 (2014) 20-27.

5. H. L. Yin and Y. J. Xu, RP-HPLC determination of strontium ranelate and its related substances, Chin. J. Pharm. Anal. 2 (2009) 330-333.

6. A. V. Gajbhar, V. P. Choudhari and B. S. Kuchekar, Development and validation of a HPTLC method for determination of strontium ranelate in the presence of its impurities, Int. J. Pharm. Bio. Sci. 6 (2015) 386-394.

7. A. S. Swami, S. A. Pishawikar and H. N. More, UV-spectrophotometric method development and validation for estimation of strontium ranelate in bulk, Int. J. Pharm. Bio. Sci. 3 (2012) 171-176.

8. M. Rizk, M. M. A. El-Alamin and M. I. Moawad, A simple kinetic spectrophotometric method for determination of strontium ranelate in bulk and pharmaceutical dosage form, Indo Am. J. Pharm. Res. 5 (2015) 2503-2512; https://doi.org/10.1044/1980-iajpr.150701

9. R. C. de Carvalho, A. D. P. Netto and F. F. de Carvalho Marques, Simultaneous determination of strontium ranelate and aspartame in pharmaceutical formulation for the treatment of postmenopausal osteoporosis by capillary zone electrophoresis, Microchem. J. 117 (2014) 214-219; https://doi. org/10.1016/j.microc.2014.06.024

10. B. V. Srinivas, U. V. Prasad, M. L. N. Acharyulua and T. S. Reddy, Determination of strontium ranelate in pure and pharmaceutical formulations by oxidimetry, Anal. Chem. Indian J. 13 (2013) 205-209.

11. M. Rizk, M. M. A. El-Alamin, H. A. M. Hendawy and M. I. Moawad, Highly sensitive differential pulse and square wave voltammetric methods for determination of strontium ranelate in bulk and pharmaceutical dosage form, Electroanalysis 28 (2016) 770-777; https://doi.org/10.1002/ elan.201500437

12. Z. I. Szabó, B. Székely-Szentmiklósi, B. Deák, I. Székely-Szentmiklósi, B. Kovács, K. Zöldi and E. Sipos, Study of the effect of formulation variables on the characteristics of combination tablets containing enalapril maleate and indapamide as active substances using experimental design, Acta Pharm. 66 (2016) 191-206; https://doi.org/10.1515/acph-2016-0019

13. A. Gavan, A. Porfire, C. Marina and I. Tomuta, Formulation and pharmaceutical development of quetiapine fumarate sustained release matrix tablets using a QbD approach, Acta Pharm. 67 (2017) 53-70; https://doi.org/10.1515/acph-2017-0009

14. A. M. Kashid, D. A. Ghorpade, P. P. Toranmal and S. C. Dhawale, Development and validation of reversed phase HPLC method for the determination of vildagliptin using an experimental design, J. Anal. Chem. 70 (2015) 510-515; https://doi.org/10.1134/S1061934815040061

15. L. Kumar, M. S. Reddy, R. S. Managuli and P. K. Girish, Full factorial design for optimization, development and validation of HPLC method to determine valsartan in nanoparticles, Saudi Pharm. J. 23 (2015) 549-555; https://doi.org/10.1016/j.jsps.2015.02.001 
16. M. Carcu-Dobrin, M. Budau, G. Hancu, L. Gagyi, A. Rusu and H. Kelemen, Enantioselective analysis of fluoxetine in pharmaceutical formulations by capillary zone electrophoresis, Saudi Pharm. J. 25 (2017) 397-403; https://doi.org/10.1016/j.jsps.2016.09.007

17. L. Eriksson, E. Johansson, N. Kettaneh-Wold, C. Wilkström and S. Wold, Design of Experiments Principles and Applications, $3^{\text {rd }}$ ed., MKS Umetrics AB, Umeå 2008.

18. User guide to MODDE, MKS Umetrics AB, Malmö 2014; http://umetrics.com/sites/default/files/ downloads/1/user_guide_to_modde_10.1.pdf; last access date November 10, 2017.

19. R. Peraman, K. Bhadraya and Y. P. Reddy, Analytical quality by design: a tool for regulatory flexibility and robust analytics, Int. J. Anal. Chem. 2 (2015) 1-9; https://doi.org/10.1155/2015/868727

20. International Conference on Harmonisation of Technical Requirements for Registration of Pharmaceuticals for Human Use, ICH Harmonised Tripartite Guideline, Pharmaceutical Development Q8 (R2), Step 4, ICH, August 2009; https://www.ich.org/fileadmin/Public_Web_Site/ICH_Products/ Guidelines/Quality/Q8_R1/Step4/Q8_R2_Guideline.pdf; last access date: February 20, 2017.

21. P. K. Sahu, N. R. Ramisetti, T. Cecchi, S. Swain, C. S. Patro and J. Panda, An overview of experimental designs in HPLC method development and validation, J. Pharm. Biomed. Anal. 147 (2018) 590611; https://doi.org/10.1016/j.jpba.2017.05.006

22. M. Marusteri and V. Bacarea, Comparing groups for statistical differences: how to choose the right statistical test? Biochem. Med. 20 (2010) 15-32; https://doi.org/10.11613/BM.2010.004 